\title{
Open Access - eine elektronischen Umgebungen angemessene Institutionalisierungsform für das Gemeingut „Wissen“
}

Open Access - institutionalizing knowledge as a commons in electronic environments

Rainer Kuhlen

August 2010

\section{PREPRINT}

Artikel erscheint online

unter der folgenden DOI 10.1007/s11578-010-0097-3

in gedruckter Version in:

Leviathan - Berlin Zeitschrift für Sozialwissenschaft 2010

[von der Website des Autors frei zugänglich in der eingereichten Textversion - zu zitieren aus der publizierten Online- oder gedruckten Verlagsversion]

Schlüsselwörter: Geistiges Eigentum; Gemeingüter; Informationsmärkte; Marktversagen; Open Access, Selbstregulierung; Tragik der Gemeingüter; Urheberrecht; Wissenschaftsfreiheit

Keywords: Open access; commons; commoners; copyright; freedom of science; information markets; intellectual property; market failure; self regulation; tragedy of the commons

Zusammenfassung: Open Access wird als angemessene Institutionalisierungsform für das Gemeingut "Wissen" angesehen. Sowohl die monopolistisch organisierten Informationsmärkte als auch das Urheberrecht erweisen sich heute eher als störende denn als fördernde Faktoren für einen in der Wissenschaft erforderlichen freizügigen Umgang mit Wissen und Information.

Abstract: Open access is an appropriate means for institutionalizing knowledge as a commons in electronic environments. Both monopolistic commercial information markets and copyright regulations are disabling rather than enabling factors in the open and free access to knowledge and information that is necessary in science and education. 


\section{Erweiterte Zusammenfassung}

Open Access wird nicht nur als eine elektronischen Umgebungen angemessene Publikationsform für Wissens- und Kulturobjekte angesehen, sondern auch eine im Sinne der Institutionenökonomik angemessene Institutionalisierungsform für das Gemeingut (Commons) „Wissen“. In der Auseinandersetzung mit der Hardin'schen These der „tragedy of the commons" wird deren Allgemeingültigkeit bestritten, vor allem auch, dass Privatisierung und damit einhergehend Kommerzialisierung oder staatliche Regulierung die einzigen angemessen Formen der Rettung der Gemeingüter vor Zerstörung seien. Es werden Selbstregulierungsmechanismen der von dem Gemeingut Profitierenden ins Spiel gebracht. Sowohl die Entwicklungen auf den internationalen Publikationsmärkten mit starken Monopoltendenzen und entsprechenden Marktverzerrungen als auch die seit gut 20 Jahren die kommerzielle Verwertung unterstützenden staatlichen Regulierungsmaßnahmen über das Urheberrecht erweisen sich heute eher als störende („disabling“) denn als fördernde („Enabling“) Faktoren für einen in Bildung und Wissenschaft erforderlichen freizügigen Umgang mit Wissen und Information. Zum Ende werden mehr Fragen gestellt als Antworten bezüglich der sich abzeichnenden Transformationsprozesse in elektronischen Räumen gegeben. Für klassische Konzepte wie Wissenschaftsfreiheit, geistiges Eigentum, Autor oder Werk müssen neue Konsense gefunden werden. Open Access hat alle Chancen zu einem Paradigma für den Umgang mit Wissen und Information zu werden und damit eine allgemeine Anerkennung von Wissen als Gemeingut zu befördern.

\section{Eine kurze Beschreibung des Open Access-Modells}

Der freie Zugang zu Internetseiten, der „Open Access“ (OA) bedeutet einen Paradigmenwechsel bei den Verbreitungsformen im Wissenschaftsbetrieb: Bei Open Access ist die Nutzung von öffentlich gemachtem und mit öffentlichen Mitteln produziertem Wissen aus Bildung und Wissenschaft grundsätzlich für jedermann frei. „Frei“ heißt hier zunächst „,frei in den Nutzungs- und Weiterverarbeitungsformen", aber durchaus auch 
„gebührenfrei: Weder die Nutzer noch die Vermittlungsorganisationen (etwa die Bibliotheken) zahlen für die Nutzung von publiziertem Wissen, wie im kommerziellen Verwertungsmodell üblich. Die Kosten, die im Publikationsprozess anfallen, werden entweder von den Produzenten (also den Autoren selber) oder von deren Institutionen getragen, oder die Anbieter bzw. Vermittler von Informationsgütern kommen für die Kosten in der Publikations- und Distributionskette auf. Das bedeutet im Falle der kommerziellen Anbieter (i. d. R. Verlage), sofern sie sich für das Open Access-Modell entscheiden, dass ihre Geschäftskonzepte sich nicht auf Einnahmen aus Verkauf oder Lizenzierung der Wissensvermittlung finanzieren lassen, sondern dass andere Finanzierungsformen gefunden werden müssen. Die US-Informationsfirma Google hat exemplarisch vorgemacht bzw. Anregungen gegeben, wie im sogenannten ökonomiefreien Modell (Freeconomics-Modell) dennoch Geld verdient werden kann: Die Information selbst ist frei, verdient wird mit Anderem, zum Beispiel mit Werbung oder mit dem Verkauf der durch die Nutzer implizit bereitgestellten Daten. Trotz erster Ansätze ${ }^{1}$ sind von Seiten der Informationswirtschaft noch kaum Modelle zu erkennen, die das Paradigma des freien Zugangs grundsätzlich anerkennen. Die Wirtschaft setzt bislang weiter auf Verkauf oder Lizenz ihrer Informationsgüter.

Die für Open Access verbindliche "Berlin Declaration" von $2003^{2}$ legt unmissverständlich fest, was darunter zu verstehen ist: „diese Veröffentlichungen

- in jedem beliebigen digitalen Medium und für jeden verantwortbaren Zweck - zu kopieren, zu nutzen, zu verbreiten, zu übertragen und öffentlich

1 Der Springer-Verlag lässt in seinem Open-Choice-Modell (http://www.springer.com/dal/home/open+choice?SGWID=1-40359-0-0-0) den Autoren die Wahl, wie bisher nach dem traditionellen Publikationsmodell dem Verlag seinen Artikel mit exklusiven Verwertungsrechten zu überlassen oder aber den Artikel frei nach dem OA-Modell verfügbar machen zu lassen. Im letzteren Fall behält der Autor alle seine Rechte an dem Beitrag, muss allerdings dafür einen Betrag von ca. \$2300 (derzeit Euro 2.000) an den Verlag entrichten.

2 Die Berliner Erklärung knüpft direkt an die Budapest Open Access Initiative von 2001 an (http://www.soros.org/openaccess/read.shtml), ebenso an die ECHO (European Cultural Heritage Online)-Charta (http://echo.mpiwg-berlin.mpg.de/home/documents/charter) und die ebenfalls 2003 verabschiedete Bethesda-Erklärung (Bethesda Statement on Open Access Publishing http://www.earlham.edu/ peters/fos/bethesda.htm). 
wiederzugeben sowie Bearbeitungen davon zu erstellen und zu verbreiten, sofern die Urheberschaft korrekt angegeben wird..

In der Urheberrechtssprache heißt das, dass Autoren, sofern sie sich für Open Access entscheiden, die ihnen zustehenden Verwertungsrechte als einfache Nutzungsrechte der Öffentlichkeit geben - was im übrigen nicht ausschließt, dass sie ihre Werke trotzdem weiter kommerziell verwerten. Allerdings können sie, wenn sie sich für Open Access entschieden haben, ihre Rechte nicht mehr als exklusive Nutzungsrechte an kommerzielle Verwerter geben. Die Konsequenzen sind für verwertende Organisationen ähnlich. Sofern sie sich für Open Access entscheiden, geben sie ihre vertraglich erworbenen Nutzungsrechte als einfache Rechte an die Öffentlichkeit weiter - was im Übrigen auch nicht ausschließt, dass sie ihre Rechte für kommerzielle Zwecke weiter nutzen. Erfahrungen von verschiedenen Verlagen deuten daraufhin, dass die Freigabe der elektronischen Versionen von Volltexten den Verkauf der gedruckten Bücher keineswegs mindert ${ }^{3}$.

Open Access ist nicht mit der Art von Selbstpublikation gleichzusetzen, wie sie durch das World Wide Web praktisch für jeden möglich geworden ist ${ }^{4}$, sondern ordnet sich in die qualitätssichernde Praxis der Wissenschaft ein. Das gilt in erster Linie für das, was nach einem Vorschlag von Stevan Harnad $^{5}$ "the golden road" genannt wird, also die Publikation in speziellen

3 Dies deutet auch darauf hin, dass das Open Access-Modell, das bislang eher auf Zeitschriftenpublikationen beschränkt war, sich auf Volltexte ausweitet. Exemplarisch hierfür sei das Telota-Vorhaben der Berlin-Brandenburgischen Akademie der Wissenschaften erwähnt. Die Politik der Akademie zielt darauf ab, die Volltexte in der elektronischen Version über einen Publikationsserver frei ins Web zu stellen und gibt verschiedenen Verlagen die Möglichkeit, gedruckte Versionen zu erstellen und kommerziell zu vermarkten. Bedeutsam bei dieser Politik ist zudem, dass es der Akademie durch eine einfache Änderung der Arbeits- und Werkverträge der Mitarbeiter gelungen ist, die Urheber zu einer Übertragung ihrer Rechte an der Verwertung ihrer Publikationen an die Akademie zu veranlassen (vgl. Andermann 2007).

${ }^{4}$ Die Form der Selbstpublikation, also das Publizieren des Autors selber ohne vorausgegangene Qualitätsabschätzung durch andere, soll hiermit keineswegs abgewertet werden, zumal dann nicht, wenn sie mit kollaborativen Verfahren verbunden ist, wie es am Beispiel der Wikipedia im großen Stil praktiziert wird. In elektronischen Räumen entwickeln sich zudem neue Formen der öffentlichen Bereitstellung von Wissen, aber auch neue Formen der Bewertung der Qualität von Arbeiten, z.B. indem die Fachwelt (und zwar jedermann) durch ihre Reaktion auf öffentlich bereitgestellte Arbeiten die Einschätzung vornimmt und nicht nur die etablierten Experten im Peer Reviewing.

${ }^{5}:$ http://www.nature.com/nature/focus/accessdebate/21.html; vgl. (Harnad et al. 2004) 
Open Access-Zeitschriften, deren Qualität genauso wie bei bisherigen Zeitschriften von den Herausgebern bzw. den Editorial boards und den Reviewern bzw. den Reviewing-Verfahren abhängt ${ }^{6}$ und für die entsprechende Finanzierungsformen gefunden werden müssen. Open Access-Publizieren ist nicht zum Nulltarif zu haben.

Bei dem anderen Open Access-Weg, "the green road", werden Texte, die zur Publikation angenommen oder schon publiziert sind, in offene Archive (Open Archives) - auch Repositorien (Institutional Repositories) genannt ${ }^{7}$ eingespeist und frei zugänglich gemacht ${ }^{8}$. Die Qualitätssicherung ist unproblematisch, wenn, im Sinne einer Zweitverwertung, die für eine Veröffentlichung nach einem Gutachterverfahren angenommenen Arbeiten in Repositorien aufgenommen werden. Dies kann zeitgleich oder, wie bislang eher üblich, mit einer gewissen Verzögerung (Embargo-Frist genannt) geschehen. Aus Zitationsgründen wäre es wünschenswert, wenn die Aufnahme in das Repositorium in der Publikationsversion des Verlages geschähe. Tatsächlich gestehen Verlage (so z.B. Elsevier) i.d.R. nur die Aufnahme in der Originalversion des Autors zu.

Weltweit wird derzeit die Diskussion darüber geführt, inwieweit erzwungen (required) oder nur nahegelegt (requested) werden soll, dass Forschungsergebnisse, die mit Fördermitteln unterstützt produziert wurden, zumindest über den grünen Weg, also als Zweitpublikation, öffentlich zugänglich gemacht werden. Weitergehend sind Überlegungen, jeden Wissenschaftler in mit Steuermitteln finanzierten Instituten zu dieser Zweitveröffentlichung nach dem grünen Weg zu veranlassen. Umstritten ist, ob „veranlassen“ auch hier als „required“ oder als „requested“ interpretiert

6 OA-Zeitschriften werden über das Directory of Open Access Journals nachgewiesen: http://www.doaj.org/. Juli 2010 waren 5160 OA-Zeitschriften aufgeführt.

${ }^{7}$ Repositorien können weltweit gesucht werden über http://opendoar.org/find.php.

${ }^{8}$ In diese Repositorien, die z.B. von den Hochschulen oder außeruniversitären Forschungseinrichtungen bzw. deren Bibliotheken betrieben werden, könnten sicher aber auch andere Werke aus der jeweiligen Institution eingestellt werden, einschließlich der zugrundeliegenden Materialien, wie experimentelle und statistische Daten oder multimediale Teile. Daher sollte auch in den nach dem grünen Weg verfahrenden Institutional repositories eine klare Kennzeichnung in den Metadaten der von den Wissenschaftlern ihrer Einrichtung erstellten Veröffentlichungen vorgenommen werden, aus denen die für die Einschätzung der Qualität und Herkunft der Arbeiten wichtigen Informationen entnommen werden können. 
werden soll. Gesetzliche Regelungen zugunsten des „required“ gibt es z.B. in den USA. Auch die EU experimentiert im Rahmen ihres 7. Forschungsförderprogramms mit Möglichkeiten, das „required“ durchzusetzen. Die DFG in Deutschland beschränkt sich derzeit auf das „requested“. Private Förderinstitutionen, wie der Wellcome Trust in England können leichter das „requested“ erzwingen.

\section{Open Access im Kontext der weltweiten Debatte über Gemeingüter}

Vom Konzept her ist die zu Beginn angegebene Spezifizierung „mit öffentlichen Mitteln" nicht zwingend. Auch Unternehmen, in deren Forschungs- und Entwicklungsabteilungen Wissen produziert wird, können selbstverständlich ihre Wissenserzeugnisse nach Open Access-Prinzipien öffentlich zugänglich machen. Und sie sollten es vermehrt tun - sind sie doch die Nutznießer der mit öffentlichen Mitteln erstellten und nach Open Access freigestellten Werke. Open Access spezifiziert nicht den Typ des Nutzers. Wenn etwas Open Access gestellt wird, ist es für jedermann frei zugänglich und für jeden verantwortbaren Zweck nutzbar.

Ebenso ist für Open Access die Beschränkung auf Bildung und Wissenschaft nicht konstitutiv. Die Berlin Declaration spricht in ihrem Titel zwar von "Open Access to Knowledge in the Sciences and Humanities", fordert aber in ihrem Deklarationstext verschiedentlich auch die Bewahrungsinstitutionen von Kulturgut (,holders of cultural heritage“) auf, sich dem Open AccessModell anzuschließen.

Auch wenn wir uns im Folgenden in erster Linie auf das in Bildung und Wissenschaft produzierte Wissen beziehen werden, ist der Hinweis auf die umfassendere Bedeutung von Open Access doch wichtig, da nur durch diese umfassende Betrachtung der Paradigmenwechsel deutlich werden kann, der durch die weltweite wissenschaftliche Debatte über Gemeingüter (Commons) angestoßen wurde (z.B. Barnes 2008; Benkler 2006; Boyle 2008; Ostrom 1990/1999) und Eingang in die zivilgesellschaftliche Diskussion gefunden hat (z.B. Bollier 2008; The Commons Rising 2006; State of the Commons 2008; Helfrich 2009). In Deutschland wird dieser Wechsel unter dem Begriff der Gemeingüter bzw. der Wissensallmende diskutiert (Netzwerk freies Wissen 2009; Helfrich et al. 2010). Diese Debatte ist nicht zuletzt dadurch in das öffentliche Bewusstsein gerückt, als 2009 der 
Commons-Forscherin Elinor Ostrom der Wirtschaftsnobelpreis zugesprochen wurde.

Wir wollen zeigen, dass die Open Access-Prinzipien mehr sind als "nur" eine alternative Form des Publizierens - dies ist wichtig genug -, sondern vielmehr als Hinweise zu deuten sind, dass der Umgang mit Wissen und Information in elektronischen Umgebungen nicht überwiegend von kommerziellen Interessen gesteuert sein muss bzw. nicht gesteuert sein sollte und dass Selbstregulierungsformen der Wissen und Information Produzierenden und Nutzenden in vielen Situationen zu besseren, Innovationen ermöglichenden Lösungen führen als staatliche Regulierungsformen (das ist in unserem Fall in erster Linie das Urheberrecht). Dabei werden wir keinesfalls die Selbstregulierungsformen als privatwirtschaftliches Handeln oder staatliche Regulierung ersetzend ansehen. Ähnlich dem Vorschlag von Peter Barnes (2008) wird aber durch die Initiativen der von den Gemeingütern Profitierenden ${ }^{9}$ - in unserem Fall sind das für das Gemeingut „Wissen“ die Wissensproduzenten und Wissensnutzer - sozusagen eine Dritte Säule, neben (nicht gegen) Wirtschaft/Markt und Staat, für die Nutzung, Bewahrung und Förderung von Wissen eingeführt.

Beispiele für die Realität dieser Dritten Säule gibt es auch in elektronischen Umgebungen für immaterielle Gemeingüter ${ }^{10}$. Muster ist seit vielen Jahren die Sicht auf Software als frei und offen nutzbare. Nicht die freie (kostenfreie) Nutzung ist hierbei entscheidend, sondern die Freiheit, die öffentlich bereitgestellte Software als offen nutzen, also selbständig weiterentwickeln zu dürfen bzw. die Verpflichtung, die Weiterentwicklungen ebenfalls offen zugänglich zu machen. „Frei“ ist nach der immer wieder zitierten Formulierung von Richard Stallman nicht im Sinne von „Freibier" zu verstehen, sondern als Fähigkeit, selbstbestimmt mit Software umgehen zu können und vor allem auch die Freiheit zu haben,

\footnotetext{
${ }^{9}$ Linebaugh (2008) hat dafür den Begriff der Commoners eingeführt.

10 In der Literatur (s. oben) werden überwiegend Beispiele für von den "Commoners" eingesetzten Regulierungsformen für natürliche Commons angeführt, z.B. energieautonome Dörfer, lokale Wasserwirtschaften, Initiativen zur Gestaltung des öffentlichen Raums (z.B. public gardens) etc.
} 
mit anderen zusammenarbeiten zu dürfen ${ }^{11}$. In der Öffentlichkeit ist für die Selbstorganisation von Wissen durch die "Commoners" selber sicherlich die Wikipedia das bekannteste Beispiel. Auch hier sind freie und offene Nutzung und kollaborative Wissensproduktion die wesentlichen Merkmale, welche die Zuordnung zum Commons-/Gemeingüter Gedanken nahelegen.

Open Access in der Wissenschaftskommunikation ist ein wichtiger Baustein für diese Dritte Säule - wichtig und unverzichtbar vor allem deshalb, weil ganz offensichtlich weder die Marktmechanismen noch staatliche Regulierungsformen (wie das Urheberrecht) dem ausreichend Rechnung tragen, dass der offene und freizügige Austausch von publiziertem Wissen für Bildung und Wissenschaft konstitutiv ist und damit im Interesse der gesamten Gesellschaft liegt. Man kann es auch drastischer ausdrücken: Sowohl Marktversagen als auch Politikversagen - beides nicht zuletzt auch verursacht durch eine unzureichende Berücksichtigung der Potenziale moderner Informations- und Kommunikationstechnologien und -methodik - haben gewissermaßen Open Access mit Notwendigkeit hervorgebracht und damit auch eine Rückbesinnung auf den Charakter von Wissen, einmal in die Welt gesetzt, als immaterielles Gemeingut bewirkt.

\section{Sollten wir von einer Tragödie der Commons oder besser vom Drama der Commons sprechen?}

Wir kommen später auf die These des Markt- bzw. Politikversagens zurück, wollen uns aber vorab noch mit der von Garrett Hardin 1968 aufgestellten These von der "tragedy of the commons" (Tragik/Tragödie der Gemeingüter) auseinandersetzen. Denn tatsächlich werden noch immer die von Hardin aus der These gezogenen Schlussfolgerungen als ideologischer Unterbau für die Überführung der Gemeingüter in privatwirtschaftliche und/oder staatliche Regulierung verwendet.

Hardins These wurde in erster Linie am Beispiel der Umwandlung des öffentlichen Weidelandes in privaten Großgrundbesitz in England seit dem 16. Jahrhundert entwickelt. Hardin behauptete, dass Gemeingüter, sich selbst überlassen, zwangsläufig zerstört würden. Für Hardin schien es

\footnotetext{
11 'nterview mit Richard Stallman „Freie Software ist kein Freibier" http://www.taz.de/1/zukunft/konsum/artikel/1/freie-software-ist-keinfreibier/?src=TE\&cHash=fa41136fff
} 
unvermeidbar, dass gemeinsames, scheinbar nicht weiter kontrolliertes Weideland übernutzt werde. Es gäbe immer Menschen, die zur Sicherung eines kurzfristigen Gewinns mehr Schafe auf die Weide brächten, als diese langfristig ertragen kann. Übernutzung (auch durch stetig anwachsende Bevölkerung in der bürgerlichen Gesellschaft der Neuzeit) und Trittbrettfahrer (free-rider) als egoistisches Durchsetzen eigener Interessen gegenüber Gruppen- oder Allgemeinheitsinteressen werden von ihm als die wesentlichen Ursachen für die Tragik der Gemeingüter ausgemacht.

Der einzige Ausweg aus dieser Tragik der Gemeingüter - und dieser Vorschlag war folgenreicher als die eigentliche These der Tragik - bestehe darin, dass die Nutzung der Gemeingüter privat oder staatlich reguliert würde. Die als vogelfrei angesehenen Gemeingüter müssten also in privates Eigentum oder staatlich kontrolliertes öffentliches Eigentum überführt würden. Durch diese regulative Überführung werde die Nutzung der Gemeingüter über entsprechende Verknappungsformen so eingeschränkt, dass sie auch langfristig erhalten bliebe. Dieses Argument bekam später auch dadurch einen gewissen Charme, als es mit dem Prinzip der ökologischen Nachhaltigkeit im Einklang zu sein schien.

Die private Aneignung der vormals gemeinschaftlich genutzten Weiden durch Großgrundbesitzer wird auch „first enclosure“ (Einhegung, Einzäunung) genannt und hat in der Theorie der politischen Ökonomie eine besondere Bedeutung erlangt (vgl. dazu Karl Marx, Das Kapital Bd. I, Max Weber, Wirtschaftsgeschichte, Karl Polanyi, „The Great Transformation“). Sie ist jedoch, anders als Hardin es darstellt, kein zwangsläufig sich ergebender Ausweg aus einer Tragödie, sondern ist ein historischer Vorgang in der kapitalistischen Entwicklung Englands. Eine Verallgemeinerung derart, dass Gemeingüter stets und zwangsläufig auf eine tragische Entwicklung hinausliefen, ist unzulässig. .

Uns interessiert hier natürlich weniger die Weidetragik als die Frage, welche Folgen die von Boyle (2003) sogenannte "second enclosure“ hat, also die heutige private Aneignung (Einzäunung) des immateriellen Gemeinguts „Wissen“. Löst diese Einzäunung die Gefahr der Tragik dieses Gemeinguts oder ist vielmehr diese Lösung heute das eigentliche Problem? Aber zurück noch einmal zu Hardin. 
Die These und die Folgerungen daraus beruhen weniger auf umfassenden empirischen Untersuchungen als auf einem Menschenbild, das sich auf die Vorstellung eines egoistisch handelnden homo oeconomicus gründet. Demgegenüber hat Ostrom in ihren durch weltweite Feldstudien gestützten Arbeiten, überwiegend mit Blick auf natürliche Gemeingüter wie Wasserversorgung, Fischerei oder Weideland, überzeugend nachgewiesen (Ostrom 1990/1999; Ostrom et al. 2002; Ostrom et al. 1994), dass die von Hardin aufgestellte These der "tragedy of the commons" keineswegs generell gültig ist.

Ostrom konnte nachweisen, dass es Menschen durchaus gelingt, für die gemeinschaftliche Nutzung einer Gemeingutressource („common-pool resource") Regeln und Durchsetzungsmaßnahmen (Institutionalisierungsformen) zu entwickeln, wodurch diese Ressource als gemeinschaftliches Gut, eben als Gemeingut, durch jeden genutzt werden kann, aber nur in dem Maße, in dem es als Gemeingut für die Gemeinschaft und für zukünftige Generationen erhalten bleibt.

Für das Gemeingut Wasser bedeutet das z.B. nach dem "reparian principle“ des angelsächsischen „common law“, dass jeder für seinen Gebrauch Wasser entnehmen kann, aber nur insoweit genug für alle anderen und in unverminderter Qualität übrig bleibt. Das steht auch mit dem Locke'schen Eigentumsbegriff im Einklang. Menschen sind durchaus in der Lage, im kommunikativen Austausch kreative Lösungen für die Nutzung und Bewahrung der Gemeingüter zu finden, und zwar durchaus auch solche, die kurzfristige Gewinnmitnahmen und Partikularinteressen zugunsten zukünftiger Generationen hinten anstellen. Um die Terminologie des Justinianischen Rechts aufzugreifen - Gemeingüter sind keine res nullius, Güter, die niemandem gehören, wie Hardin offenbar ursprünglich annahm $^{12}$, keine vogelfreien Güter, vielmehr sind sie res communes, die historisch durch Einzäunung zu res privatae gemacht worden sind.

Mit dieser Einsicht hatte das „Committee on the Human Dimension of Global Change" 2001 einen Sammelband mit dem Titel „The Drama of the Commons" vorgelegt (Ostrom et al. 2001). Durch die Ersetzung von

\footnotetext{
12 In (Helfrich et al. 2010, 17) wird darauf hingewiesen, dass Hardin später seinen 1968er Aufsatz eher als „Analyse der Tragik der unverwalteten Gemeingüter" verstanden haben wollte.
} 
„Tragödie“ durch „Drama“ (Schauspiel) im Titel des Buches sollte auf den offenen Ausgang von Geschichte hingewiesen werden: Das Schauspiel der Geschichte muss nicht immer als Tragödie enden, sondern kann ebenso einen guten Ausgang nehmen. Im Sammelband werden auf breiter Basis Beispiele gegeben, dass der Umgang mit den Gemeingütern auch zum glücklichen Ende eines Schauspiels führen kann, wenn man die Gemeinschaft der „Commoners" nur lässt.

Der Coomons-Forschung ist auch zu entnehmen, dass es für den Umgang mit den Gemeingütern keine universale Institutionalisierungsform gibt. Auch ist es nicht immer die Gemeinschaft selber, die die Lösung finden kann. An dem Gemeingut Luft bzw. Klima wird deutlich, dass hier nur überstaatliche Regulierungsmaßnahmen die wirkliche Tragödie der Welt mit dramatischem Klimawandel vermeiden können. Es sind auch sicher Beispiele anzuführen, bei denen der Markt geglückte Lösungen für die Gemeingüter entwickelt hat.

Wie beim Theater, überwiegen aber bislang mit Blick auf die Gemeingüter die tragischen Entwicklungen. Bei vielen, auch elementaren natürlichen Gemeingütern scheint die Tragik der Vernichtung oder zumindest der weitgehenden Verletzung bedrohlich bevorzustehen: Wasserwirtschaft. Fische in den Meeren und Flüssen, die Luft nach vielen Jahren der unkontrollierten Zufuhr von Industrie- und Verbraucherschadstoffen sind Beispiele solcher sich ankündigender Tragödien. Gesundheitswesen, Finanzwesen oder der öffentliche Raum entwickeln sich als soziale Gemeingüter kaum mehr im Interesse der davon unmittelbar Betroffenen. Auch sie drohen tragisch zu kollabieren.

Dass viele der ökologischen und ökonomischen Probleme der Gegenwart nicht zuletzt durch Übernutzung oder gar Zerstörung der natürlichen Gemeingüter entstanden sind, das dürfte heute kaum mehr auf Widerspruch stoßen - Externalisierung von Kosten gilt betriebswirtschaftlich sogar als Wettbewerbsvorteil. Dass aber auch eine Unternutzung von immateriellen Gemeingütern auf Grund erzwungener Verknappungsmaßnahmen ebenfalls höchst schädliche Folgen haben kann, ist eher noch ein wissensökologisch und wissensökonomisch ungewohntes Argument (Kuhlen 2004). 
Damit sind wir zum eigentlichen Gegenstand dieses Beitrags zurückgekommen. Open Access ist ganz im Sinne der Institutionenökonomik als eine Institutionalisierungsform für die Gemeingutressource „Wissen“ anzusehen. So wie andere natürliche Ressourcen erst durch entsprechende Institutionalisierungsformen zu Gemeingütern (oder auch zu privaten Gütern) werden, so entscheidet sich auch durch die Institutionalisierungsform für immaterielle Gemeingüter, ob sie tatsächlich als Gemeingüter oder ob sie als Verwertungsobjekte für den kommerziellen privaten Handel betrachtet und durchgesetzt werden können. Gemeingüter sind naturgegeben, aber ihre Nutzung wird im Prozess des Aushandelns und des Interessenausgleichs bzw. der Machtdurchsetzung sozial reguliert.

Betrachtet man das heutige Ergebnis der Institutionalisierung des Umgangs mit Wissen und Information, so kann man, zumindest aus der Sicht der Wissenschaftlerinnen und Wissenschaftler, kaum anders als zu dem folgenden Schluss kommen: Die in den letzten gut 20 Jahren entwickelten Marktmechanismen für den Umgang mit immateriellen Gemeingütern (Wissens- und Kulturobjekten), aber auch die diese Entwicklung in erster Linie über das Urheberrecht begünstigende staatliche Regulierung haben eine immer stärker werdende Verknappung der Nutzung bewirkt. Auf Verknappung setzende Märkte und ein starkes, die Verwertung begünstigendes Urheberrecht erweisen sich heute immer mehr als störende („Disabling-“), kaum noch als fördernde („Enabling-“) Faktoren für Wissensfortschritt und innovative Anwendungen von Wissen in der Wirtschaft.

Man ist also versucht, gegen Hardins Lösungsvorschlag die Gegenthese aufzustellen, dass die private Aneignung der Gemeingüter durch kommerzielle Interessen ebenso die Gemeingüter bedrohen wie auch eine zu starke staatliche Regulierung, die häufig genug auch eher zugunsten der Wirtschaftsinteressen eingreift, und nicht unbedingt zugunsten der Nutzer der Gemeingüter. Diese These, gekoppelt mit dem Vorwurf sowohl des Markt- als auch Staatsversagens, kann hier nicht weiter ausgeführt werden ${ }^{13}$. Stattdessen werden in den folgenden beiden Abschnitten nur

${ }^{13}$ Vgl. insbesondere Benkler 2006; Castells 2009; Hansen 2009; Hilty 2006,2007; Kreutzer 2008; Kuhlen 2008; Nentwich 2003. 
einige Hinweise gegeben, dass zum einen die Fehlentwicklungen auf den Märkten die Entwicklung von Open Access hervorgebracht haben und dass zum andern das (bisherige) Scheitern aller Bemühungen aus den Bereichen Bildung und Wissenschaft, gegenüber dem Gesetzgeber ein wissenschaftsfreundlicheres Urheberrecht anzumahnen, als ein erfolgreiches Scheitern gedeutet werden kann (Kuhlen 2008): Mehr und mehr Wissenschaftler erkennen, dass sie nicht länger warten können, bis Markt und staatliche Regulierung die notwendigen Auswege aus den von innen geschaffenen Aporien gefunden haben, sondern dass sie, nicht gegen Markt und Staat, aber doch selbstbestimmt Open AccessPublikationsformen und freie Lizenzierungsformen wie „Creative Commons “14 anwenden müssen.

Wir wollen zum Abschluss fragen, inwieweit der sich in elektronischen Umgebungen entwickelnde freizügige und teilende Umgang mit Wissen und Information nicht nur neue Open Access-Publikationsformen begünstigt, sondern auch, inwieweit traditionelle, unserer Gesellschaft zugrundeliegende Konzepte wie Wissenschaft, Wissenschaftsfreiheit, geistiges Eigentum, Autor oder Werk transformiert werden und inwieweit diese Transformationsprozesse die Sicht auf Wissen als Gemeingut befördern werden.

\section{Marktversagen bringt Open Access hervor}

Die kommerzielle Verwertung von Wissen und Information hat durch die Öffnung des Internet für die Privatwirtschaft seit Mitte der 90er Jahre stark zugenommen. Hier sind auf den Märkten einige Verschiebungen zu erkennen, und zwar von den im Wissenschaftsbetrieb direkt involvierten traditionellen Verlagen, die mit persönlichem Engagement und einem quasi wissenschaftlichen Ethos ihr Geschäft betreiben (wie in vielen mittelständischen Verlagen in Deutschland üblich), zu heute immer mehr global agierenden Akteuren. Letztere sind (als Aktiengesellschaften) dem wissenschaftlichen Publikationsgeschehen kaum mehr durch Tradition und Verpflichtung verbunden, sondern betrachten auch wissenschaftliche

\footnotetext{
${ }^{14}$ Creative Commons (CC) ist eine Non-Profit-Organisation, die in Form vorgefertigter Lizenzverträge eine Hilfestellung für die Veröffentlichung und Verbreitung digitaler Medieninhalte anbietet. (http://de.creativecommons.org/was-ist-cc/)
} 
Information in erster Linie unter dem Verwertungsaspekt und der Aussicht auf Gewinnmaximierung. Hier sind es vor allem die für Wissenschaft zentralen Zeitschriftenmärkte, auf denen hohe Umsätze und Gewinne erzielt werden, vor allem wenn die Portfolios der anbietenden Verlage so umfangreich und Monopolrechte gesichert sind, dass Verkauf oder Lizenz quasi zu einem Selbstläufer werden. Übrig geblieben sind in diesem Wettbewerb weltweit im Grunde nur noch vier große Anbieter: Reed Elsevier:PLC, Wiley Interscience, Thompson Publishing Group Inc. und Springer Science+Busines Media.

Dass die private Informationswirtschaft sich seit gut 20 Jahren exklusive Rechte an den in Bildung und Wissenschaft erstellten Werken sichert, scheint zunächst wenig mit der Hardin'schen Vermutung zu tun zu haben, dass die Privatisierung und die damit einhergehende Kommerzialisierung den Weg zur Rettung von Gemeingütern weisen. Die immateriellen Gemeingüter waren bei Hardin ohnehin nicht im Blick. Insofern könnte man die Hardin'sche These ganz zu den Akten legen, zumal seine Tragödie sich auf Übernutzung gründete.

Die „Wissensallmende“ (Netzwerk freies Wissen 2009) ist ja durchaus etwas ganz anderes als die Allmende der Schafweiden. Wissen und Information sind keine Gemeingüter, die durch Übernutzung vernichtet werden können. Informationen als immaterielle Gemeingüter erfahren anders als die natürlichen Gemeingüter durch Weitergabe und häufige Nutzung nicht automatisch einen Wertverlust. Ganz im Gegenteil - je mehr sie genutzt werden, umso größeren Nutzen stiften sie. Free-Riding, also die Nutzung von publiziertem Wissen, ohne dafür Kompensationsleistungen zu erbringen, kann in einer volkswirtschaftlichen Gesamtrechnung durchaus positive Effekte haben (Lemley 2004).

Nein, nicht Rettung des Gemeinguts „Wissen“, sondern die Aussicht auf erhebliche Gewinnmargen hat die professionelle Informationswirtschaft in die wissenschaftlichen, zunehmend elektronisch organisierten Informationsmärkte einsteigen lassen. Sie profitieren alle von traumhaften Vorleistungen der Öffentlichkeit, wie sie sonst wohl kaum in einem Wirtschaftszweig anzutreffen sind: Die Forschungsumgebung, einschließlich der Personalkosten der Forscher und ihrer Teams sowie der technischen Infrastruktur, wird von der Öffentlichkeit bereitgestellt. Öffentlich 
finanzierte Wissenschaftler fassen ihre Forschungsergebnisse in Artikeln zusammen, oft schon unter Beachtung der für die Verlagsveröffentlichung vorgesehenen Formatierung. Die Begutachtung der Qualität der Arbeiten, die peer-review, stützt sich auf die Kompetenz anderer Wissenschaftler, die nicht bezahlt werden müssen. Die Autoren geben i.d.R. alle ihre im Urheberrecht garantierten Verwertungsrechte an ihren Artikeln als Nutzungsrechte an die Verlage ab, so dass sie mit diesen zunächst als Zeitschriftenbeiträge vorgesehenen Werken in jeder medialen Form handeln dürfen. Für diese Übertragung der Rechte erhalten in der Regel weder die Autoren noch deren Institutionen irgendwelche monetäre Kompensation. Am Ende der Kette muss die Öffentlichkeit, überwiegend über die Bibliotheken, die ursprünglich von ihr finanzierten Wissensgüter nun als publizierte Informationsprodukte zurückkaufen oder über den Kauf von Lizenzrechten deren Nutzung ermöglichen ${ }^{15}$.

Dieses Modell hatte Erfolg - von den großen Konsortien wurde in den letzten Jahren durchweg Renditen um die $20 \%$ und mehr erzielt ${ }^{16}$, auch deshalb weil wissenschaftliche Zeitschriftenmärkte Monopolstrukturen aufweisen (was Verleger, Zeitschriften und einzelne Artikel angeht), so dass Preise für Zeitschriften nicht über den Markt über Angebot und Nachfrage gesteuert wurden, sondern fast nach Belieben mit ständig ansteigenden Abonnementspreisen festgesetzt wurden. Dass Bibliotheken bei diesen Preissteigerungen nicht mitmachen konnten und laufend Zeitschriften, schließlich auch Kernzeitschriften verschiedener Fachgebiete abbestellen mussten, hat jeder Nutzer in den letzten Jahren erfahren. Unterstützungen wie die Nationallizenzen, die die DFG den Verlagen abkaufte und den Bibliotheken zur Verfügung stellte, haben nur teilweise geholfen ${ }^{17}$. Immer

15 Natürlich sei nicht verschwiegen und nicht unterschätzt, dass Verlage nach dem Erwerb der Nutzungsrechte auch Mehrwertleistungen, z.B. durch Organisation der Begutachtung, der Textaufbereitung, des Erstellens von Volltextdatenbanken, Suchmaschinen, Marketing und Vertrieb erbringen, die, sofern sie wirkliche informationelle Mehrwerte für die Nutzer darstellen, den kommerziellen Ertrag allerdings nur teilweise rechtfertigen.

${ }^{16}$ Nur ein Beleg vom Marktführer Reed Elsevier:PLC: Umsatz 20096.071 Mio f - Gewinn nach Steuern 982 Mio f - http://www.mediadb.eu/datenbanken/internationale-medienkonzerne/reed-elsevier-plc.html. Dieser Umsatz nähert sich dem Gesamtumsatz des deutschen Buchmarktes an http://www.zeit.de/2009/16/C-digitales-Publizieren.

${ }^{17}$ Vgl. http://www.nationallizenzen.de/ 
mehr Nutzer, vor allem Studierende ohne größere finanzielle Ressourcen, greifen immer mehr auf die im Internet frei verfügbaren, aber durchaus nicht immer qualitativ gesicherten Informationsgüter zurück. Man spricht von der "Googlerisierung" der Ausbildung (Kuhlen 2005).

Das ist das, was verkürzt die Zeitschriftenkrise bzw. die Bibliothekskrise genannt wird. Eigentlich ist es eine Krise der wissenschaftlichen Informationsmärkte, eine Krise der Verlage, die an ihren aus der analogen Welt stammenden Geschäftsmodelle festhalten, die sich im Wesentlichen an der Anzahl der verkauften bzw. lizenzierten Objekte orientieren bzw. an den direkten Einnahmen aus diesen Objekten.

\section{Unintendierte Folgen: Das Urheberrecht hat die kommerzielle Verwertung begünstigt und dabei Open Access hervorgebracht}

Die zunehmende gesamtwirtschaftliche Bedeutung elektronisch vermittelten Märkte hat die staatlichen und überstaatlichen Regulierungsinstanzen seit gut 20 Jahren veranlasst, die Gesetze und Verträge zur Sicherung dessen, was geistiges Eigentum genannt wird, einer weitgehenden Revision zu unterziehen. Dass diese Revision in Richtung einer Förderung der kommerziellen Verwertung und ihrer technischen Sicherung ging, ist deutlich erkennbar.

Die Bedeutung der elektronischen Wissens- und Informationsmärkte für die Gesamtgesellschaft ist die treibende Kraft hinter supranationalen Verträgen wie dem TRIPS-Abkommen (Trade-Related Aspects of Intellectual Property Rights) der WTO oder den 96er Verträgen der WIPO (World Intellectual Property Organization), der UN-Organisation für geistiges Eigentum, aber auch hinter der EU-Richtlinie von 2001 und den entsprechenden nationalen Implementationen. Staatliche Regulierung in Form einer Stärkung des Urheberrechts, so die offizielle politische Argumentation, sei Grundlage allgemeiner wirtschaftlicher Prosperität, welche nur gegeben ist, wenn einerseits weiterhin ausreichend neues Wissen produziert und andererseits, wenn den informationswirtschaftlich operierenden Unternehmen ausreichende Anreize gegeben werden, Wissen in nutzbare Informationsprodukte und -dienste umzusetzen.

Insofern könnte man diese Argumentation durchaus in die Hardinschen Schlussfolgerungen einordnen. Tragödien des Wissens ereignen sich zwar 
nicht durch verbrauchende Übernutzung, aber tragische Entwicklungen können sich auch beim Gemeingut „Wissen“ ergeben, wenn a) das entstandene Wissen nicht öffentlich zugänglich gemacht wird und b) wenn auf Grund mangelnder Anreize nur noch unzureichend neues Wissen produziert wird - unzureichend nicht zuletzt für den Bedarf der Wirtschaft, aus neuem Wissen innovative Produkte und Verfahrensformen abzuleiten.

In der Tat ist beides ja die zentrale Rechtfertigung für den urheberrechtlichen Schutz: Den verwertenden Organisationen, so die herrschende politische Annahme, muss Rechtsschutz für die Ausübung ihrer durch Vertrag von den Urhebern erworbenen Nutzungsrechte gegeben werden, weil sonst nicht mehr in ausreichendem Maße für das Publizieren von Wissen investiert würde. Und den Urhebern muss ebenfalls Rechtsschutz als Anreiz zur Produktion neuen Wissens garantiert werden, wobei dieser Rechtsschutz sich nicht nur auf die Garantie der Persönlichkeitsrechte beschränkt ${ }^{18}$, sondern auch auf die der Verwertungsrechte ${ }^{19}$ mit der Zusicherung einer angemessenen Vergütung. Also sind doch Markt und Staat die Garanten für die Sicherung des Gemeinguts „Wissen“?

Es könnte so sein, aber es ist nicht so, wie ich an anderer Stelle ausgeführt habe (vgl. Kuhlen 2008). Sowohl in Teilen der Rechtswissenschaft ${ }^{20}$ als auch von Seiten der Interessenvertretung des Aktionsbündnisses „Urheberrecht für Bildung und Wissenschaft ${ }^{\prime 21}$, in der Zivilgesellschaft ${ }^{22}$ und in der Allianz

${ }^{18}$ Entsprechend $\S \S 12-14$ des Gesetzes über Urheberrecht ... sind dies das Veröffentlichungsrecht, die Anerkennung der Urheberschaft und der Schutz vor Entstellung des Werkes.

19 Entsprechend $\S \S 16-24$ des Gesetzes über Urheberrecht ... sind dies (neben einigen anderen): Vervielfältigungsrecht, Verbreitungsrecht, Recht der öffentlichen Zugänglichmachung (mit Letzterem ist die Veröffentlichung im Online-Medium gemeint).

${ }^{20}$ Z.B. Kreutzer 2008,2009, Hansen 2009, Hilty 2006,2007

21 Das Aktionsbündnis „Urheberrecht für Bildung und Wissenschaft“ (http://www.urheberrechtsbuendnis.de/) setzt sich für ein ausgewogenes Urheberrecht ein und fordert für alle, die zum Zweck von Bildung und Wissenschaft im öffentlichen Raum tätig sind, den freien Zugang zur weltweiten Information zu jeder Zeit von jedem Ort. Die dem Aktionsbündnisses zugrundeliegende Göttinger Erklärung wurde unterzeichnet von sechs Mitgliedern der Allianz der Wissenschaftsorganisationen, von über 365 wissenschaftlichen Fachgesellschaften, Informationseinrichtungen und Verbänden sowie von mehr als 7.200 Einzelpersönlichkeiten. 
der Wissenschaftsorganisationen ${ }^{23}$ wurde kritisiert, dass die seit 2003 erfolgten Anpassungen des Urheberrechts an die Vorgaben der EURichtlinie von 2001 den in elektronischen Räumen entwickelten Anforderungen (vgl. Castells 2009, Nentwich 2003) kaum gerecht werden. Exemplarisch sei ein noch sehr vorsichtig gehaltenes Zitat der Allianz der Wissenschaftsorganisationen zur aktuell (2010) begonnenen Dritten Anpassung des Urheberrechts in Deutschland angegeben:

„Der wissenschaftliche Arbeitsalltag, der mittlerweile weitgehend von der Digitalisierung bestimmt wird, wurde durch die bisherigen Novellen des Urheberrechtsgesetzes leider in seinen rechtlichen Rahmenbedingungen erheblich verkompliziert. Auch tragen gerade diejenigen Vorschriften, die sich mit digital vorliegenden Inhalten befassen, zu wenig den Erfordernissen einer schnellen und ungehinderten Wissenschaftskommunikation Rechnung. Dies ist innovationshemmend für den Wissenschaftsstandort Deutschland“ (Allianz o.J. 2009).

Angesichts dieser Skepsis gegenüber den bisherigen Anpassungen des Urheberrechts an die Bedürfnisse von Bildung und Wissenschaft, aber auch angesichts der Dysfunktionalität der bisherigen Marktstrukturen des wissenschaftlichen Publikationswesens, ist es nicht verwunderlich, dass sich speziell das Interesse der öffentlichen Bibliotheken beziehungsweise der großen Wissenschaftsorganisationen darauf richtete, Alternativen auszuloten, die in der analogen Publikationswelt nicht gegeben waren. Dass

22 Exemplarisch sei hier nur verwiesen auf: Lars Fischer (Hauptpetent) Petition: Wissenschaft und Forschung - Kostenloser Erwerb wissenschaftlicher Publikationen vom 20.10.2009 (https://epetitionen.bundestag.de/index.php?action=petition\%3bsa=details\%3bpetition=7922). Dazu gibt es eine Zusatzpetition durch das Aktionsbündnis Urheberrecht für Bildung und Wissenschaft: „Urheberrechte von wissenschaftlichen Autorinnen und Autoren stärken und Open Access befördern - Ergebnisse von mit öffentlichen Mitteln geförderter Forschung kostenfrei zugänglich machen“ Zusatzpetition zur Petition von Lars http://www.urheberrechtsbuendnis.de/docs/Aktionsbuendnis-petition-open-access-ergz-zuLarsFischer250110.pdf. Beide Petitionen werden vom Bundestag zusammen behandelt.

23 Die Allianz der Wissenschaftsorganisationen umfasst: Alexander von Humboldt-Stiftung, Deutscher Akademischer Austauschdienst, Deutsche Akademie der Naturforscher Leopoldina Nationale Akademie der Wissenschaften, Deutsche Forschungsgemeinschaft, Fraunhofer Gesellschaft, HelmholtzGemeinschaft Deutscher Forschungszentren, Hochschulrektorenkonferenz, Leibniz-Gemeinschaft, MaxPlanck-Gesellschaft, Wissenschaftsrat. Vgl. Stellungnahme: Urheberrecht für Bildung und Wissenschaft. Zentrale Forderungen der Allianz der Wissenschaftsorganisationen ...April 2005 http://www.hrk.de/de/download/dateien/Allianz_Position_Urheberrecht.pdf. 
dabei das Open Access-Modell auch politisch interessant wurde, ist ebenfalls nicht verwunderlich - kann doch darin, ganz abgesehen von den gesamtwirtschaftlich positiven Folgen einer freien und damit breiteren Nutzung der mit öffentlichen Mitteln produzierten Forschung, ein großes Potenzial der Kostenreduktion gesehen werden.

Daher hat auch der Deutsche Bundesrat 2006 einen Vorschlag aus der Wissenschaft aufgegriffen (Hansen 2006), nach dem es dem Urheber eines Werkes, das im Rahmen einer mit öffentlichen Mitteln finanzierten Forschung entstanden ist, grundsätzlich erlaubt sein sollte, auch bei einem vertraglich einem Verlag eingeräumten ausschließlichen Nutzungsrecht seinen Beitrag selber oder durch Dritte nach sechs Monaten ohne weiteres Entgelt an den Vertragspartner öffentlich zugänglich zu machen, etwa über ein institutionelles Repositorium - allerdings nicht zu kommerziellen Zwecken. Entsprechend hatte der Bundesrat der Bundesregierung bzw. dem Deutschen Bundestag vorgeschlagen, den § 38 UrhG entsprechend so zu ändern und vor allem eine Klausel dabei vorzusehen, dass dieses Recht der Autoren nicht über eine vertragliche Vereinbarung mit den Verlagen abbedungen werden kann (vgl. Heckmann/Weber 2006).

Der Bundesrat hat angesichts der fatalen Folgen der sogenannten Zeitschriftenkrise, die die Länder als Finanzierer der Hochschulen bzw. deren Bibliotheken unmittelbar betrifft, Handlungsbedarf der Regulierungsinstanzen gesehen, der durch eine Anpassung der bisherigen urhebervertragsrechtlichen Regelung gedeckt werden könnte ${ }^{24}$. Es gehe nicht an, dass das Fehlen einer entsprechenden Regelung „den Rechteinhabern eine weit gehend unbeschränkte Verhandlungsmacht einräumt und wissenschaftliche Autoren dazu veranlasst, jede für sie auch noch so ungünstige Vereinbarung zu unterzeichnen." Politisch wird diese Initiative der Länderkammer damit begründet, "dass den Hochschulen nach $\S 2$ Abs. 7 des deutschen Hochschulrahmengesetzes sowie nach den

${ }^{24}$ Der Bundesrat beschränkte seinen Vorschlag auf Beiträge in Zeitschriften, bezog nicht Beiträge in Sammelwerken explizit ein. Der jetzige $\S 38$ hat aber dem über Absatz 2 Rechnung getragen. Auch Artikel in nicht periodisch erscheinenden Sammelwerken (z.B. Konferenzproceedings) sollen in die Verfügung der Autoren (bislang nach 12 Monaten) zurückfallen. Dies könnte genauso für den Bundesratsvorschlag in einem entsprechenden Absatz zur Anwendung kommen. Auf jeden Fall sollen Konferenzproceedings in dieser Hinsicht den Zeitschriften gleichgestellt werden. 
einschlägigen Regelungen in den Hochschulgesetzen der Länder auch die Aufgabe des Wissenstransfers übertragen ist. Daher haben die Unterhaltsträger der Hochschulen und Forschungseinrichtungen ein elementares Interesse daran, die mit erheblichem Einsatz von Steuergeldern generierten wissenschaftlichen Erkenntnisse einer breiten wissenschaftlichen Öffentlichkeit zugänglich zu machen“. Der Bundesrat versteht seine Empfehlung, den § 38 UrhG zu erweitern, durchaus als „Paradigmenwechsel im Bereich wissenschaftlicher Veröffentlichungen an Hochschulen und Forschungseinrichtungen, der für einen möglichst freien Zugang zu wissenschaftlichen Informationen die geeigneten rechtlichen Rahmenbedingungen schafft". Zugleich sieht der Bundesrat durch die Vorschrift, dass das vorgesehene Recht der Urheber nicht abbedungen werden kann, eine Korrektur der jetzigen Schieflage zwischen den Verwertungsansprüchen der Verlage und der genuinen grundrechtlich geschützten Rechtspositionen der Wissenschaftler. Allerdings soll der Bundesratsvorschlag nur für die aus öffentlichen Mitteln finanzierten Wissenschaftler gelten. Die Bundesregierung bzw. der Bundestag sind bislang diesem Vorstoß nicht gefolgt. Im 2010 beginnenden Dritten Korb der Urheberrechtsanpassung wird die Frage nach Open Access-Regelungen im Urheberrecht erneut behandelt.

\section{Open Access und die Folgen - mehr Frage als Antworten}

Open Access - das war unsere Ausgangsthese - ist mehr als „nur" eine alternative Publikationsform für Wissens- und Kulturobjekte, bislang in erster Linie in Bildung und Wissenschaft. Open Access ist eine elektronischen Medien angemessene Institutionalisierungsform für das Gemeingut „Wissen“ so, wie Open Source ein Leitparadigma für Software in Wirtschaft und Gesellschaft geworden ist. Die Bedeutung von Open Access wird sich in Zukunft noch ausweiten, wenn, entsprechend dem Ziel des freien Zugangs zu Internetdateien (Open Data), nicht mehr nur die textuellen Repräsentationen von Wissen frei gestellt werden, sondern auch die der Forschung oft zugrundeliegenden experimentellen und empirisch ermittelten statistischen Daten. Ebenso ist zu erwarten, dass in den Öffnungsprozess noch stärker als bisher das Umfeld, der Kontext der bislang eher isoliert gesehenen Werke in das Publikationsgeschehen einbezogen werden: Erste Ideen, Vorversionen, Besprechungen, Kommentare, 
Diskussionen in elektronischen Kommunikationsdiensten wie Foren oder Blogs, Fortschreibungen der Ausgangsarbeiten in kollaborativen Diensten wie Wikis, ... Wir stellen dazu zum Abschluss nur einige Fragen, die zu beantworten zu versuchen weit über die Darstellung in diesem Rahmen hinausgehen würde:

Werden die kommerziellen Informationsanbieter in der Lage sein, Geschäftsmodelle zu entwickeln, die grundsätzlich das Open AccessParadigma anerkennen und mit denen sie sich trotzdem auf den Informationsmärkten behaupten können?

- Wer wird in Zukunft für die Kosten des Open Access-Publizierens aufkommen? Lohnt sich das Open Access-Publizieren volkswirtschaftlich?

Soll die staatliche Regulierung überhaupt in das Open AccessPublizieren eingreifen, oder sollte dies den "Commoners", also den Wissenschaftler und Nutzern selber, über Selbstregulierungsmaßnahmen überlassen bleiben?

$>$ Gehört zur grundgesetzlich garantierten Wissenschaftsfreiheit auch jede Ausprägung der sogenannten positiven Publikationsfreiheit? Unbestritten ist, dass das "ob", „wann" und „wie" des zu Publizierenden Teil der Wissenschafts-/Publikationsfreiheit ist. Gehört aber auch das „wo" dazu, also das exklusive Recht auf die Wahl des Publikationsorgans?

Hat die Öffentlichkeit das Recht, von jedem Wissenschaftler in öffentlicher Anstellung zumindest eine Zweitveröffentlichung seiner Werke in einem frei zugänglichen Repositorium zu verlangen, parallel zu einer Veröffentlichung in einem Verlag seiner Wahl?

Reichen zur Begründung des Schutzanspruchs der Urheber in Bildung und Wissenschaft die Persönlichkeitsrechte aus? Muss einem Urheber in öffentlichen Umgebungen der Anspruch auf finanzielle Vergütungsansprüche im Urheberrecht garantiert sein? Sind direkte finanzielle Belohnungen die entscheidenden stimulierenden Anreize für Wissenschaftler? 
$>$ Ist es möglich - vergleichbar mit der Änderung des Patentierungsrechts für Wissenschaftler in Deutschland ${ }^{25}$-, durch eine einfache Veränderung der Arbeitsverträge von Wissenschaftlern eine Zweitveröffentlichungspflicht zu erreichen, so wie es offenbar die Berlin-Brandenburgischen Akademie der Wissenschaften unternommen hat (vgl. Anm. 3)?

$>$ Kann das Konzept des geistigen Eigentums, sofern dieses als persönliches Eigentum reklamiert wird, weiterhin als Grundlage für das Urheberrecht angesehen werden, wenn durch die zunehmend kollaborativ sich organisierenden Arbeitsprozesse in elektronischen Medien sowohl das klassische Verständnis des singulären Autors als auch das des geschlossenen singulären linearen Werks zugunsten kooperativer Autorenschaft und nicht-linearer hypertextueller Wissensorganisation aufgelöst werden? Macht es Sinn, von einem gemeinschaftlichen Eigentum an Wissen (und Kultur insgesamt) zu sprechen?

Könnte nicht eine Legitimationskrise für die politischen und rechtlichen Instanzen entstehen, wenn für einen immer größer werdenden Anteil der Bevölkerung ein freizügiger und partizipativer Umgang mit Wissen und Information die Norm wird, die Regulierungsvorhaben aber weiter auf Verknappungsmechanismen und auf die Unterstützung der kommerziellen Verwertung setzen?

Open Access hat alle Chancen, zu einem Paradigma in elektronischen Räumen zu werden und damit eine allgemeine Anerkennung von Wissen als Gemeingut zu befördern.

\section{Referenzen}

(Allianz, 2009): Neuregelung des Urheberrechts.Anliegen und Desiderate für einen Dritten Korb - -

http://www.allianzinitiative.de/fileadmin/user upload/Home/Desiderate fuer Dritten Korb UrhG.pdf

(Andermann 2007) Andermann, Heike: The electronic life of the academy (Telota). Die Open-Access-Strategie der Berlin-Brandenburgischen Akademie der Wissenschaften. Zeitschrift für Bibliothekswesen und Bibliographie (ZfBB), Sonderheft Open Access Heft

${ }^{25}$. http://swpat.ffii.org/papri/bmj-hochpatarbeg01/index.de.html - text 
4-5, 2007, 262-267 (auch -

http://edoc.bbaw.de/volltexte/2007/553/pdf/28W852XashQ.pdf)

(Barnes 2008) Barnes, Peter: Capitalism 3.0. A guide to reclaiming the commons http://www.capitalism3.com/downloadbuy (deutsch: Kapitalismus 3.0 - Ein Leitfaden zur Wiederaneignung der Gemeinschaftsgüter Hamburg 2008 http://boell.de/publikationen/publikationen-4819.html)

(Benkler 2006) Benkler, Yochai: The wealth of networks. How social production transforms markets and freedom. Yale University Press. New Haven, London 2006 http://cyber.law.harvard.edu/wealth of networks/Download PDFs of the book

(Berliner Erklärung 2003) Berlin declaration on open access to knowledge in the sciences and humanities - http://www.zim.mpg.de/openaccess-berlin/berlindeclaration.html deutsche Version: http://oa.mpg.de/openaccessberlin/Berliner Erklaerung dt Version 07-2006.pdf

(Bollier 2008) Bollier, David: Viral Spiral. How the Commoners Built a Digital Republic of their Own. The New Press. New York, London 2008

(Boyle 2003) Boyle, James: The second enclosure movement and the construction of the public - http://www.law.duke.edu/journals/66LCPBoyle

(Boyle 2008) Boyle, James: The public domain. Enclosing the commons of the mind. Yale University Press. New Haven, London 2008 - http://thepublicdomain.org/download

(Bundesrat 2006) Stellungnahme des Bundesrates. Entwurf eines Zweiten Gesetzes zur Regelung des Urheberrechts in der Informationsgesellschaft. Drucksache 257/06 (Beschluss) 19.05.06

(Castells 2009) Castells, Manuel: Communication power. Oxford/New York: Oxford University Press

(The Commons Rising 2006) - http://www.onthecommons.org/content.php?id=1547

(EU 2001) Richtlinie 2001/29/EG des Europäischen Parlaments und des Rates vom 22. Mai 2001 zur Harmonisierung bestimmter Aspekte des Urheberrechts und der verwandten Schutzrechte in der Informationsgesellschaft. Amtsblatt Nr. L 167 vom 22/06/2001, 0010 - 0019 - download: http://eurlex.europa.eu/LexUriServ/LexUriServ.do?uri=OJ:L:2001:167:0010:0019:DE:PDF

(Hansen 2006) Hansen, Gerd: Urheberrecht für Wissenschaftler - Risiken und Chancen der Urheberrechtsreform für das wissenschaftliche Publizieren. In: Maximilian Stempfhuber (Hrsg.): In die Zukunft publizieren. Herausforderungen an das Publizieren und die Informationsversorgung in den Wissenschaften. Tagungsband zur 11. Jahrestagung der luK-Initiative der Wissenschaftlichen Fachgesellschaften in Deutschland. Bonn 2006, 9-19 - http://www.univerlag.uni-goettingen.de/univerlagDateien/veranstaltungen/hansen UrhRfWissen IUK2005.pdf 
(Hansen 2009) Hansen, Gerd: Warum Urheberrecht? Die Rechtfertigung des Urheberrechts unter besonderer Berücksichtigung des Nutzerschutzes. Schriftenreihe zu Bildung und Kultur Band 4. Nomos Verlagsgesellschaft, Baden-Baden 2009

(Hardin 1968) Hardin, Garrett: The tragedy of the commons. Science, Vol. 162, 1968, no. 3859, 1243 - 1248 - http://www.sciencemag.org/cgi/content/full/162/3859/1243

(Harnad et al. 2004) Harnad, S., Brody, T., Vallieres, F., Carr, L., Hitchcock, S., Gingras, Y, Oppenheim, C., Stamerjohanns, H., \& Hilf, E. :The access/impact problem and the green and gold roads to Open Access. Serials Review 30. http://www.ecs.soton.ac.uk/ harnad/Temp/impact.html

(Heckmann/Weber 2006) Heckmann, Jörn; Weber, Marc Philipp:: Open Access in der Informationsgesellschaft - § 38 UrhG de lege ferenda. GRUR Int 2006 Heft 12, $996 \mathrm{ff}$ (Helfrich 2009) Helfrich, Silke und Heinrich-Böll-Stiftung (Hrsg.): Wem gehört die Welt? Zur Wiederentdeckung der Gemeingüter. oekom Verlag, München 2009 - download: http://commonsblog.files.wordpress.com/2008/08/netzausgabe_wem_gehrt_die_welt_2. pdf

(Helfrich et al. 2010) Gemeingüter: Wohlstand durch Teilen. Ein Report von Silke Helfrich, Rainer Kuhlen, Wolfgang Sachs und Christian Siefkes. Herausgegeben von der Heinrich-Böll-Stiftung - download: www.boell.de/downloads/Gemeingueter Report Commons.pdf

(Hilty 2006) (Hilty 2006a) Hilty, Reto M.: Das Urheberrecht und der Wissenschaftler. GRUR -Zeitschrift der Deutschen Vereinigung für gewerblichen Rechtsschutz und Urheberrecht 55, 3/2006, 179-190

(Hilty 2007) Vgl. Reto Hilty: Sündenbock Urheberrecht? In: Ohly, A. / D. Klippel (Hrsg.): Geistiges Eigentum und Gemeinfreiheit, Bd. 11 der Schriftenreihe: Geistiges Eigentum und Wettbewerbsrecht. Tübingen, Mohr Siebeck, 2007, S. 107 - 144

(Kreutzer 2008) Kreutzer, Till: Das Modell des deutschen Urheberrechts und Regelungsalternativen. Konzeptionelle Überlegungen zu Werkbegriff, Zuordnung, Umfang und Dauer des Urheberrechts als Reaktion auf den urheberrechtlichen Funktionswandel., Nomos Verlagsgesellschaft, Baden-Baden 2008

(Kreutzer 2009) Kreutzer, Till: Den gordischen Knoten durchschlagen - Ideen für ein neues Urheberrechtskonzept. In: Copy.Right.Now! Plädoyers für ein zukunftstaugliches Urheberrecht. Schriftenreihe zu Bildung und Kultur Band 4. Hrsg. von der Heinrich-BöllStiftung In Zusammenarbeit mit iRights.info. Berlin April 2010, 45-55 - download aus: http://irights.info/blog/arbeit2.0/wp-content/uploads/2010/04/copyrightnow.pdf

(Kuhlen 2004) Kuhlen, Rainer: Wissensökologie. In: Rainer Kuhlen; Thomas Seeger; Dietmar Strauch (Hrsg.): Grundlagen der praktischen Information und Dokumentation. K.G. Saur: München, 5. Auflage 2004, 105-113 - download: http://www.kuhlen.name/MATERIALIEN/Publikationen2004/a08-kuhlen-AA.pdf 
(Kuhlen 2005) Kuhlen, Rainer: Macht Google autonom? Zur Ambivalenz informationeller Autonomie. In: K. Lehmann; M. Schetsche (Hrsg.): Die Google-Gesellschaft. Wissen im 21. Jahrhundert, transcript-Verlag 2005, 385-393 - download: http://www.kuhlen.name/MATERIALIEN/Publikationen2005/googlenachwort final100205.pdf

(Kuhlen 2008) Kuhlen, Rainer: Erfolgreiches Scheitern - eine Götterdämmerung des Urheberrechts? Schriften zur Informationswissenschaft; Bd. 48. vwh - Verlag Werner Hülsbusch: Boizenburg 2008 download:

http://www.kuhlen.name/MATERIALIEN/RK2008 ONLINE/files/HI48 Kuhlen Urheberre cht.pdf

(Lemley 2004) Lemley, Mark A.: Property, intellectual property, and free riding. Stanford Law School August 2004 - http://ssrn.com/abstract=582602

(Linebaugh 2008) Linebaugh, Peter: The Magna Carta manifesto: Liberties and commons for all. Berkeley: University of California Press, 2008

(Nentwich 2003) Nentwich, Michael: Cyberscience. Research in the age of the Internet. Austrian Academy of Sciences Press. Vienna 2003

(Netzwerk freies Wissen 2009) (Hrsg.) Wem gehört das Wissen der Welt. Wissensallmende Report 2009 (Redaktion Petra Buhr und Silke Helfrich). www.wissensallmende.de Dezember 2008

(Ostrom 1990/1999) Ostrom, Elinor: Governing the commons: The evolution of institutions for collective action. New York: Cambridge University Press 1990 (deutsch: Die Verfassung der Allmende. Jenseits von Staat und Markt 1999)

(Ostrom et al. 2002) Ostrom, Elinor et al. (eds.): The drama of the commons. National Academy Press. Washington 2002

(Ostrom et al.1994) Ostrom, Elinor, Walker, J. \& Gardner, R.: Rules, games, and commonpool resources. Ann Arbor: University of Michigan Press 1994

(State of the Commons 2008) Report and outlook - http://ecoplan.org/library/2008.pdf 